\title{
TELEREHABILITATION TECHNOLOGIES: ACCESSIBILITY AND USABILITY
}

\author{
MICHAEL PRAMUKA ${ }^{1} \&$ LINDA VAN ROOSMALEN $^{1}$ \\ ${ }^{1}$ DEPARTMENT OF REHABILITATION SCIENCE AND TECHNOLOGY, \\ UNIVERSITY OF PITTSBURGH
}

\begin{abstract}
In the fields of telehealth and telemedicine, phone and/or video technologies are key to the successful provision of services such as remote monitoring and visits. How do these technologies affect service accessibility, effectiveness, quality, and usefulness when applied to rehabilitation services in the field of telerehabilitation? To answer this question, we provide a overview of the complex network of available technologies and discuss how they link to rehabilitation applications, services, and practices as well as to the telerehabilitation end-user.

This white paper will first present the numerous professional considerations that shape the use of technology in rehabilitation service and set it somewhat apart from telemedicine. It will then provide an overview of concepts essential to usability analysis; present a summary of various telerehabilitation technologies and their strengths and limitations, and consider how the technologies interface with end users' clinical needs for service accessibility, effectiveness, quality, and usefulness. The paper will highlight a conceptual framework (including task analyses and usability issues) that underlies a functional match between telerehabilitation technologies, clinical applications, and end-usercapabilities for telerehabilitation purposes. Finally, we will discuss pragmatic issues related to user integration of telerehabilitation technology versus traditional face-to-face approaches.
\end{abstract}

Key Words: Remote, Technology, Usability, Accessibility, Decision Factors, Decision Support.

\section{INTRODUCTION}

There is a growing literature base on the use of technology for remote assessment and intervention in medicine (Bashur, 2002) and in rehabilitation (Winters, 2002). However, most descriptions and projects are limited to one or two types of technology and one population. Applications are often described for the field of medicine without exploring the unique history and needs of rehabilitation service delivery that set it apart from medicine. In addition, many rehabilitation providers function outside of formal hospital or medical settings and may not be aware of all of the technology options for telerehabilitation. These issues present obstacles for the agency, individual provider, and consumer who would like to consider implementing telerehabilitation for a particular environment, purpose, or disability group that may not match either available descriptions or what they know/ currently access and use.
For telerehabilitation to best benefit the end-user (i.e., an individual with a disability) within their environment, all stakeholders need access to the greatest possible set of options to determine what telerehabilitation service delivery system will yield the best results. Moreover, as telerehabilitation services continue to supplement or replace traditional face-to-face clinical services, there is an increasing need to standardize the clinical practice, reimbursement, and health care policies that apply to telerehabilitation. 


\section{OVERVIEW OF PROFESSIONAL CONSIDERATIONS FOR TELEREHABILITATION}

Numerous challenges arise when rehabilitation services are provided remotely instead of in traditional face-to-face contexts. Many of these challenges set telerehabilitation apart from the broader use of telemedicine and telehealth. Theodorus \& Russell (2008) provided a summary of potential barriers to the implementation of telerehabilitation, namely, licensure laws, professional issues, reimbursement, and characteristics of disability. We will comment on these challenges and examine the historical differences between the provision and reimbursement opportunities for acute vs. community re-entry services; the need for working contracts; the importance of ongoing contact with family and friends; and disability adjustment issues related to technology.

Telemedicine's models and successes should not be assumed to automatically transfer to the field of telerehabilitation. Although a subset of rehabilitation services is delivered in acute or post-acute medical settings by licensed professionals, many rehabilitation services occur in other settings and are supported by the funding streams that reside outside the traditional medical setting and medical/diagnostic billing systems. Reimbursement and professional services for individuals seeking services for independent living, vocational rehabilitation, residential services, or other community re-entry functions are not typically funded by medical insurance plans. These costs have always, even in face-to-face rehabilitation settings, been addressed by an amalgam of county funding, state funding, state/ federal vocational funds, grants, and other sources. Stakeholders seeking reimbursement for telerehabilitation thus find themselves mired in a maze of non-profit and government-based organizations. Similarly, many community level services are provided by workers who are not allied with a specific professional background or licensure, in part because the funding streams are not adequate to support salaries for licensed professionals. Sadly, it is common for individuals with a significant acquired disability to expend all of their financial resources from medical insurance on acute and postacute medical services, only to find themselves without financial support when their level of recovery finally reaches a stage at which they are ready to resume independent function or explore paths to re-employment.

In addition, many individuals experience a disability and seek rehabilitation services due to injury or illness linked to their employment. Rehabilitation services for these individuals are provided under worker's compensation, and are linked more to the structural etiology of the disability (i.e., the employment experience) than to specific diagnoses. Funds under these plans may be able to pay for a variety of both acute and community re-entry services, as long as the need for such services is ultimately linked back to the workplace disability.

The majority of funding for face-to-face rehabilitation services has traditionally been provided in the context of acute rehabilitation services. In contrast, the most likely funding stream for telerehabilitation reimbursement originates from high-cost professional services provided in post-acute care. The urgent need to provide community re-entry services in any manner, however, suggests that the domain of independent living, vocational rehabilitation, and psychosocial support would be an even more fruitful area for development. The myriad of funding streams and frequently less technological orientation of these community services, however, makes reimbursement more likely to occur in professionalized settings.

In rehabilitation, more so than in diagnostic medicine, the development of a working alliance or working contract, good rapport with the client, and concurrent involvement of family and friends is critical to the therapeutic process. Therapeutic relationships require a degree of trust and a willingness to take risks, and remote service provision may challenge the ability of therapists to develop rapport. Poor video connections, an inability to use touch therapeutically, a lack of a shared space, and the challenge of communicating commitment and interest via technology may make it more difficult to establish rapport. On the other hand, telerehabilitation might offer a potential advantage in drawing in the family and friends of clients given the use of technology in the home or local clinics. Telereehabilitation in the home or local community offers also the great benefit of reduced travel.

Much of the work in telemedicine and in tele-health involves the use of technology primarily by professionals. In contrast, the ongoing, therapeutic, and feedback oriented aspects of rehabilitation are much more likely to require active participation on the part of the client. Yet, many of these clients will be seeking rehabilitation services precisely because of a recent and significant change or decline in functional status. These changes may render keyboards, software, or other technology use much more challenging than it would have been for before the onset of disability. In addition, individuals tend to describe their strengths and limitations and make decisions based on their previous levels of function. Only after rehabilitation efforts are successful over time do we adapt and incorporate changes of physical or cognitive ability into our identity, and make decisions comfortably with these altered characteristics in mind.

Finally, as many have noted in professional statements and journal articles, the standards, laws, and licensure issues that have been implemented at a state level face significant challenges in interpretation and implementation for telerehabilitation services. (Koocher 2007).

Overview of Accessibility and Usability Issues for Technology 
Not all products are designed with the end-user in mind. Think about asking someone with large hands to use a cell phone with small control buttons, or consider the need to be physically present at a certain time and day of the week to record your favorite television program. Issues similar to those that we face with daily technology may be experienced by the receiver of remote clinical services. Examples of technologies used for remote service delivery include computers, internet connections, and wireless devices (cell phone, monitoring device, PDA).

Clinicians who communicate with people with disabilities and participate with them in remote rehabilitation type services will need to become knowledgeable about technology usability and accessibility. If clients are given a role in managing technology during the rehabilitation session (e.g., measuring, collecting and transmitting data) clinicians may experience difficulties related to the client's (mis) understanding and (mis)use of technology. Nursing assistants and other clinical personnel who assist clients may not be trained to provide remote rehabilitation services, and may experience similar difficulties with the use and installation of technology. Technology difficulties in the various stages of use (i.e., connecting, installing, adjusting, programming, recording/sending data etc.) can negatively affect the effectiveness of telerehabilitation.

At the University of Pittsburgh, we conduct weekly staff meetings between vocation rehabilitation specialists at two locations via a state-of-the-art video conferencing system. Videoconferencing works well because it enables everyone to see who is speaking and facilitates "turntaking." However, the set-up process involves several steps. The two group leaders turn the device on and manipulate the cameras to visually include all participants. One button controls starting the system, another controls the camera position, a different remote controls screens, and another remote can be used to start and record sessions. When dialing in, it is important to select the (no charge) IP number and not the IDSN number, because the IDSN number is associated with high connection costs. Finally, some tweaking of the camera is needed when multiple people attend the meeting. When group leaders are not present, attendees who have witnessed the setup process many times before may not be successful in completing the videoconference setup, and resort instead to use of a speakerphone.

This example clearly demonstrates that the video conferencing system is difficult to use for people who typically do not use this type of technology. The system's "usability" can be improved upon by making the installation procedure simpler for everyday use, and by creating a pre-programmed system that has a simple step-by-step interface to use, adjust, and maintain the system.

Adopting a "universal design" (UD) approach to telerehabilitation technology may solve many of the issues referred to in this manuscript. Researchers at North
Carolina State University developed seven principles of UD (Table 1). When technologies are designed according to these principles, both rehabilitation service providers and recipients may encounter less difficulty in their use and adoption (Story, 1988).

Table 1: Principles of Universal Design (North Carolina State University) 1

\section{UD Principle 1: Equitable Use}

The design is useful and marketable to people with diverse abilities.

\section{Guidelines:}

1a. Provide the same means of use for all users: identical whenever possible; equivalent when not.

1b. Avoid segregating or stigmatizing any users.

1c. Provisions for privacy, security, and safety should be equally available to all users.

1d. Make the design appealing to all users.

\section{UD Principle 2: Flexibility in Use}

The design accommodates a wide range of individual preferences and abilities.

\section{Guidelines:}

2a. Provide choice in methods of use.

$2 b$. Accommodate right- or left-handed access and use.

2c. Facilitate the user's accuracy and precision.

$2 d$. Provide adaptability to the user's pace.

\section{UD Principle 3: Simple and Intuitive Use}

Use of the design is easy to understand, regardless of the user's experience, knowledge, language skills, or current concentration level.

\section{Guidelines:}

3a. Eliminate unnecessary complexity.

3b. Be consistent with user expectations and intuition.

3c. Accommodate a wide range of literacy and language skills.

3d. Arrange information consistent with its importance.

3 e. Provide effective prompting and feedback during and after task completion.

\section{UD Principle 4: Perceptible Information}

The design communicates necessary information effectively to the user, regardless of ambient conditions or the user's sensory abilities.

\section{Guidelines:}

4a. Use different modes (pictorial, verbal, tactile) for redundant presentation of essential information.

4b. Provide adequate contrast between essential information and its surroundings.

4c. Maximize "legibility" of essential information.

4d. Differentiate elements in ways that can be described (i.e., make it easy to give instructions or directions).

$4 \mathrm{e}$. Provide compatibility with a variety of techniques or devices used by people with sensory limitations. 
UD Principle 5: Tolerance for Error

The design minimizes hazards and the adverse consequences of accidental or unintended actions.

\section{Guidelines:}

5a. Arrange elements to minimize hazards and errors: most used elements, most accessible; hazardous elements eliminated, isolated, or shielded. 5b. Provide warnings of hazards and errors.

5c. Provide fail safe features.

$5 d$. Discourage unconscious action in tasks that require vigilance.

UD Principle 6: Low Physical Effort

The design can be used efficiently and comfortably and with a minimum of fatigue.

\section{Guidelines}

6a. Allow user to maintain a neutral body position.

$6 b$. Use reasonable operating forces.

$6 c$. Minimize repetitive actions.

$6 \mathrm{~d}$. Minimize sustained physical effort.

\section{UD Principle 7: Size and Space for Approach and}

\section{Use}

Appropriate size and space is provided for approach, reach, manipulation, and use regardless of user's body size, posture, or mobility.

Guidelines

7a. Provide a clear line of sight to important elements for any seated or standing user.

7b. Make reach to all components comfortable for any seated or standing user.

7c. Accommodate variations in hand and grip size.

7d. Provide Adequate Space for the use of assistive devices or personal assistance.

These UD principles are to be used to design, improve, or make decisions for products and systems that can be used by as many people as possible. UD Principle 2 (flexibility in use) and UD Principle 3 (simple and intuitive use) are especially important when selecting and using technologies for remote clinical services. The technology chosen should to be designed for persons with diverse abilities, flexible in use, simple, and intuitive. Although this paper addresses technology design, we hope to raise awareness among clinicians and other stakeholders about the usability assessment of technology as a key component to the adoption of telerehabilitation.

\section{OVERVIEW OF TELEREHABILITATION}

\section{TECHNOLOGIES}

A wide variety of technologies are available to provide telerehabilitation services, and a growing body of literature describes the use of specific telerehabilitation technologies to address the needs of defined client populations. Here we provide both a narrative and tabular overview of technologies in current use as well as emerging technologies with application to telerehabilitation. Given the disparate technologies used to conduct tele-services, it can be challenging to outline and describe general technology options. In terms of technical specifications, the sensory modality, and the path or technology of data transmission are probably the most overt to describe and provide an easy distinction. We therefore outline technologies based on the primary sensory modality used to communicate data, with later, more detailed discussion on matching technology to clinical needs.

\section{TEXTUAL BASED TECHNOLOGIES}

Text has been the basis for shared communication across time and differing locations for hundreds of years. While the use of written reports or forms completed at home or other remote sites and then mailed in offers some improvement over required face-to-face visits, the rapid advances in technology have now provided almost universal access to more efficient, technologybased distribution and sharing of textual information. Email programs are now available at no cost, and, with the advent of wireless technologies, are no longer tied to computer use. Textual technologies allow simple recording, displaying, or streaming of textual information that can later be displayed in the form of reports and diagrams. Email exchange may serve as the basis for both professional education and ongoing therapeutic communication. Cell phone text messages can provide brief reminders or guidance to consumers. Text is asynchronous traditionally, but approaches synchrony with instant messaging.

One non-language based example of this type of data is the acceleration data from a client's wheelchair that is remotely emailed to a clinician to evaluate the overall fitness and activity level of the individual over time. Another example is a text message automatically generated to remind an individual to take medications at a specific time. 


\section{AUDIO (VOICE/SOUND) BASED TECHNOLOGIES}

A wide range of technologies exist that can convey both voice and other audio data from one location to another. The technology behind audio transmission has broadened significantly beyond traditional phone lines. Audio may now be carried via cable connections/phone lines, via voice over internet protocol (VOIP), wirelessly via cell phones (see below) or accompany videoconferenced technology over ISDN lines. Audio offers significant data and information at relatively minimal bandwidth requirements compared to visual data. In addition to these options for real-time/synchronous transmission of audio, audio may also be stored and forwarded at a future date via .wav files or other MP3 formats. Numerous devices are also now capable of digitally storing audio data for immediate replay at a future time, or for download. These digital audio recordings are easily stored electronically and can be shared or forwarded as email attachments at no cost. For those with access to existing technology, audiotapes and videotapes continue to offer the opportunity to store and forward audio at minimal cost.

Telephone use is probably the most common use of audio technology for telerehabilitation purposes, via oneto-one voice conversations, or use of a speakerphone or a teleconferencing system. The latter allows multiple parties to participate in the same real-time conversations from distance locations (e.g., family meetings with professionals; and staff participation in client/patient staffings). One caveat for TR implementation is that audio over speakerphones, cell phones, and accompanying videoconferences can only transmit one voice or location sound at a time, requiring some rehearsal and adaptation so that multiple speakers do not cut each other off when trying to use normal conversational patterns. Increasingly, however, the easy availability of podcasts using audio on iPods or other MP3 players allows information to be replayed as frequently and whenever the client chooses, offering the opportunity to listen to professional lectures on rehabilitation topics, review instructions for use of assistive technology, or even replay client self-instructions in their own voice on sequencing a task. Stored memos in voice can also serve as a memory or organizational compensatory strategy. Longer recordings of classroom lectures or meetings can be downloaded into a computer file for sharing and for review at the client's pace. Additional technologies may include a POTS, an automatic audio recording through a POTS or cell phone or a recording of educational materials for use on an I-pod, tape recorder or cd-player.

\section{VISION BASED TECHNOLOGIES}

Video may be used either in real-time (synchronously, such as videoconferences) or asynchronously (emailed photographs, for example). Real-time videoconferencing may be achieved in a wide variety of ways. Options include simple and inexpensive webcams (when the visual images can be of coarse granularity); relatively inexpensive videoconferencing equipment (when facial features or other high quality visual images or movement are important); and expensive and complex videoconferencing systems (when a broad field of vision or multiple sites are involved, such as for client staffing or professional training). For videoconferencing systems, the required bandwidth, security of transmitted images, and importance of dependability are also significant factors to consider. Videoconferencing can be conducted via the Internet on Internet Protocol (IP) using existing phone lines at no greater cost that computer internet use, or via installed, dedicated lines (ISDN) which offer much greater security and quality dependability, but also high initial installation costs as well as monthly maintenance charges and per minute usage fees. In general, the total bandwidth available will be divided by the number of sites in contact with each other. The Veterans Healthcare System has established a VA Telecommunications Modernization Project (TMP) in which the type of data is classified into voice, video, "excellent effort traffic", and "best effort traffic" and then prioritized depending on the needs of the clinical videoconference. This allows the bandwidth to be maximized for the type and quality of service needed rather than depending on quality and quantity of local data being carried (Darkins, Cruise, Armstrong, Peters, \& Finn, 2008).

Asynchronous visual data may be useful to transmit still images or photographs via a "store and forward" mode in which data is first acquired and stored and then forwarded via the internet or via cell phone at some future time. Asynchronous video may also be recorded on DVD, videotape, or cell phones and mailed or transmitted electronically. As with videoconferencing, the level of required visual integrity and the security desired will play a role in determining which modality is most appropriate.

\section{VIRTUAL REALITY}

Virtual Reality (VR) provides a unique opportunity to simultaneously present and manipulate a variety of visual, auditory, tactile/haptic, and even olfactory sensations to represent physical experiences. While there must be some minimal neuronal or sensorimotor involvement of the participant to engage them in VR, the actual experience may primarily be cognitive, social, or emotional, similar to human experiences in the physical world. In general, the spectrum of VR experiences can be characterized by the qualities of immersion and invasiveness. How immersive or engaging is the experience? Is the interface complex and what modalities does the VR experience access? As 
with any telerehabilitation effort, VR technology will be subservient to the overall clinical or educational needs being served.

Visually oriented VR can be administered without a physical interface via relatively inexpensive head-mount sets that look like goggles or via images projected on a screen. Some room sized VR environments (CAVEs) provide much greater flexibility and participant immersion but may cost over one million dollars to construct. VR with tactile/haptic feedback is available to provide training for individuals with motor impairment.

VR now also comprises internet-based sites that offer entire worlds based on computer games, but with the distinction that participants have the freedom to move about and engage each other outside of predetermined rules. Based on computer games conducted over the internet with many players (Massively Multiplayer Online Games, MMROGS), VR websites include SIMS and SecondLife.com, in which avatars engage in an internet based world, with opportunities for therapeutic guidance to virtually explore social consequences and alternative response choices.

\section{WEB-BASED TECHNOLOGY}

Websites offer a wide variety of incorporating text, audio, images, and video experiences that may incorporate both real-time communication and stored material for asynchronous use into a rehabilitation service. Website features include real-time chatrooms to connect individuals to each other for education and support; discussion boards to share information and resources; short videos; written information; and interactive assessment or educational modules. There has been a burgeoning interest in the use of websites to supplement or replace professional face-to-face intervention services for people with chronic medical conditions using a self-management or psychoeducational perspective. Websites are able to store information about previous visits, modify presentations based on user requests or user responses to short assessments, and provide prepared guidance based on user needs. Two primary examples of web-based technologies in telerehabilitation are those used to administer and monitor selfmanagement therapy programs for chronic conditions (Lorig, Ritter, Laurent, \& Plant, 2006), and continuing education programs that allow professionals to maintain or update their professional education without traveling to a conference. A third, more recent application of webbased technology is the coordination of assessment, such as that conducted in the VA/DoD for service members with brain injury. This system incorporates a variety of checklists and patient-rating scales entered by clinicians into the system. (Girard, 2007).

\section{WIRELESS TECHNOLOGIES}

The field of wireless technology offers numerous opportunities for the remote provision of rehabilitation. PDA's (Personal Digital Assistants) can be used for several functions. PDA use can enhance someone's memory and assist individuals with cognitive limitations in performing daily tasks and keeping appointments. PDA's can also serve as a tool to administer and transmit cognitive assessments, particularly in military or other remote environments where standard face-to-face administration is not feasible (Girard, 2007). The use of Global Positioning Systems (GPS) can guide visually or cognitively impaired individuals as they travel through streets or exit from buildings. Individuals can pre-visit certain environments to become comfortable with the surroundings (Google Maps). New technology via the use of accelerometers is under development to provide inside navigation for individuals with visual impairments .

Cell phones and smart phones offer the opportunity to capture a visual image of a pressure ulcer and wirelessly transmit it to a clinician who will determine the pressure ulcer stage and the needed intervention. Cell phone technology can also easily bridge distance when following up with a remote client. Podcasts and other services via iTunes such as I-Tunes University use digitally formatted material for educational purposes. With the widespread use of the iPod, digital media is increasingly accessible to a wide population.

\section{INTEGRATED SYSTEMS}

Increasingly, telerehabilitation systems depend on integrated systems that either combine multiple sensory and technical interfaces on both sides, or integrate one technology on the remote side with a different technology on the clinician/near side. Integrated systems allow users to represent a wider variety of communication media that may accurately mimic face- to-face interaction. They also support the transmission and sharing of critical components (vs. a large volume of data with minimal value added). Integrated systems will also be needed to address the complex needs associated with new and multifaceted disability such as polytrauma in veterans, which presents needs as diverse as case management, assistive technology, and home modification (Bendixen, Levy, Lutz, Horn, and Chronister, 2008). In addition, the field of robotics is now being applied to rehabilitation applications, offering opportunities to integrate haptic or visual responses with artificial intelligence and high data storage capacity (Brewer, McDowell, \& WorthenChaudhari, 2007; Carnigan \& Krebs, 2006). As telerehabilitation practice grows both in popularity and sophistication, it is likely that a greater number of systems will use this integrated systems approach to maximize communication and data exchange options. 


\section{MATCHING TECHNOLOGIES TO REHABILITATION NEEDS: USABILITY AND ACCESSIBILITY ISSUES}

The technologies that have potential to be used as telerehabilitation technologies (TTs) have been discussed in the previous section. This section will focus on the use of technologies and how various users of technologies and the clinical/rehab application will affect effective use of TTs.

Potential users of TTs include the patient, their caregivers, physicians, therapists, engineers and others. Some of these individuals are on the clinical side, whereas others are on the receiving patient side of the TT. Because there are two or more users involved with the TT the human interaction system needs to work and be usable from both sides, meaning that both rehabilitation professionals as well as clients need to be able to use the TT (Winters, 2002; Brennan, 2008). Characteristics of users will affect proper and safe use of technology.

Effective translation of traditionally provided clinical services to remote delivery requires a good understanding of the various aspects of clinical services, consumer needs and response modalities, and an accurate understanding of the limitations of technology. The choice of TT to meet the needs of service providers and the end-useressentially begins with a task analysis of the clinical (rehabilitation) task at hand and the characteristics of the potential user. Remote services and the technology that provides the infrastructure introduce a new environment into the clinical rehabilitation relationship that may differ significantly from face-to-face interaction. For example, individuals with traumatic brain injury face a combination of cognitive and physical changes that make the learning of new systems challenging due to the demands on visual memory and executive functions, both impaired in these individuals (Diamond, Shreve, Bonilla, Johnston, Morodan, \& Branneck, 2003).

Domains to consider in moving from face-to-face to remote rehabilitation include: overall goals of clinical intervention, typical timeframes of interaction, communication modalities required for clinician and consumer, modality of data to be exchanged, and usability of technology by the consumer. Frequently, careful evaluation of consumer needs and telerehabilitation technologies may suggest several alternative models of remote intervention, each with significant strengths and limitations, to be assessed with the consumer for best overall fit.

For example, TTs can range from very simple technology such as a pen and paper-based log book and a POTS (plain old telephone system) that assists cognitively impaired individuals in keeping track of daily activities and responsibilities. A more complex version of this logbook that will perform a similar function can be an electronic Personal Digital Assistant (PDA) that also keeps track of daily activities by entering in activities and responsibilities and by communicating with the therapist by digitally transmitting a summary of the weekly logbook.

Whereas the first option uses simple tools to fulfill a certain (monitoring/advising) task, the second option fulfills the same task but uses a more complex configuration of technologies and connection systems. The complex system may prevent some users from using the system properly and actually benefitting from its features.

There are a large number of clinical applications that utilize telerehabilitation technologies. Winters suggested several categories of clinical applications that may require special technologies (Table 2).

Table 2: Classification of clinical activities for remote service provision.

\section{Teleconsultation:}

A medical/legal term that suggests communication across distance in which there is an expert consultant who provides a service.

\section{Teleconferencing:}

The process of two or more people interacting across a distance, supported via telecommunications.

Telematics:

The study of intelligent, effective strategies for transporting and utilizing health-related information and/or healthcare services.

Tele-education:

The process of education/training at a distance. (Education represents one of the key professional activities of a visiting home health nurse.)

Telemonitoring (interactive):

The process of monitoring health status at a distance. Involves interactive conferencing, and perhaps the transmission of data (e.g., vital sign recordings).

Telemonitoring (unobtrusive):

The process of unobtrusive sensing of personal health status, or of the environment.

\section{Telesupport:}

The process of interactive support, e.g. via a tele-nurse. Depending on the protocol, the "patient" and/or the provider of support may initiate the call.

Tele-evaluation:

Systematic professional evaluation at a distance, e.g. by a physician, therapist, nurse, rehabilitation engineer, or other health professional.

Teleassessment:

Systematic assessment of health status by a healthcare professional, often more broad-based that an evaluation. Normally would need to be interactive.

Telediagnosis: The process of performing diagnosis at a distance. 


\section{Telecompliance:}

The process of providing support, encouragement and education at a distance, so as to enhance compliance with health maintenance (e.g., taking medication) or home selftherapy (e.g., a prescribed exercise program).

\section{Teletherapy:}

The process of actual therapeutic intervention at a distance. For instance, physical/occupational or psychological/ psychiatric therapy. Ideally, there would be built-in objective telemonitoring/teleassessment that related to performance and outcomes measures.

\section{Teleplay:}

Use of interactive, exploratory "games" with built-in therapeutic and/or monitoring capabilities, and with interface parameters that can be adjusted depending on the client's progress.

Telecoaching:

The process of trained supervisor providing proactive support, training and assessment related to helping a client improve performance and abilities.

To provide clinicians with a direction for what technology may be most effective, the type of data needed to perform the clinical application should now be matched to the required data type with a specific technology. It is also important to understand how potential users from both ends of the medium may become (informationally) handicapped when being forced to use a certain technology. The authors suggest an identification (model) of the relationship between media types that are needed to perform certain clinical applications. It is important to understand the type of media that needs to be recorded/monitored/ communicated so that the clinical application is effective for both the clinicians and the clients and their caregivers. For example, when conducting a psychological evaluation of an individual, one may think that use of the telephone may be sufficient to assess the client. However, client behavior may not be fully captured simply by recording and/or hearing the client's voice. Research shows that in addition to voice, a key factor in client assessment is viewing bodily posture and facial expression. An appropriate technology such as a voice recorder would miss important information for an appropriate client assessment, whereas video conferencing or a prerecorded video of the client will provide the necessary information to conduct a thorough assessment.

\section{Table 3. Complexity of telerehabilitation technologies.}

\section{Complexity Level 1}

Data Type: Textual

Functional Features: messages, feedback

Telerehabilitation Technology: pen or pencil and paper, text to phone, email

\section{Complexity Level 1}

Data Type: Audio

Functional Features: voice, speech, language,

communication, memory

Telerehabilitation Technology: voice recorder, phone, POTS

\section{Complexity Level 2}

Data Type: Visual (2D)

Functional Features: shape, stage, image, schematics, location

Telerehabilitation Technology: cell phone, camera

\section{Complexity Level 2}

Data Type: Time \& Textual

Functional Features: planning, monitoring data, history, improvement

Telerehabilitation Technology: planner, PDA

\section{Complexity Level 3}

Data Type: Visual (3D)

Functional Features: space, movement, motion, (bodily) fit, human-product interaction, gait, safety

Telerehabilitation Technology: video, radar, 3D camera, multiple cameras

\section{Complexity Level 4}

Data Type: Audio and Visual (3D)

Functional Features: interaction, expression, personality, user opinion

Telerehabilitation Technology: videoconferencing, digital video

\section{Complexity Level 4}

Data Type: Touch - Haptics

Functional Features: texture, resistance, temperature

Telerehabilitation Technology: sensors, gauges

\section{Complexity Level 5}

Data Type: Time and Visual (3D)

Functional Features: location, speed, acceleration

Telerehabilitation Technology: GPS, accelerometer, video, wireless

\section{Complexity Level 5}

Data Type: Time and Haptics

Functional Features: resistance, force, moment Telerehabilitation Technology: accelerometer, sensors, robotics

\section{Complexity Level 6}

Data Type: Haptics Time, Audio \& Visual (3D)

Functional Features: behavior in environment; prediction Telerehabilitation Technology: Virtual Reality; robotics, sensors and cameras 
Table 3 presents a schema of various media types and associated functional input. Six levels of complexity categorize data that is least demanding (singledimensional, simple data) to most demanding (multidimensional, complex data) to record. For example, Complexity Level 6 requires capturing or relaying haptics, time, sound, and vision data and can be accomplished by Virtual Reality (VR) or a combination of robotics, sensors and cameras. This level is the most complex and likely requires the most setup, installation and training time for users of the technology.

\section{DECISION FACTORS}

The selection of a remote rehabilitation system entails multiple decision factors. The technology must fit a clinic's budget, application purpose, and technical support structure. The technology must also match well to the skills and perceptions of the client and clinician. Very complex technology (i.e., consisting of multiple components, settings and/or connections) may be associated to higher cost and a higher learning curve, which may ultimately result in technology abandonment and non-use of the system.

\section{COMPLEXITY}

The level of complexity of technologies can have an impact on how easy technologies are in their everyday use and how much training is required to operate and adjust the technology. Complex technologies may involve several components that need to be connected together, programmed, and/or customized for specific purposes. A high level of technology complexity may require an internal or external technical support system (helpdesk) which may not be available at the remote client end.

\section{INSTALLATION}

Ease of installation is another decision factor when selecting a technology to conduct remote rehabilitation services. Is specific software needed to run the technology? Is the software easy to install by a layperson or is a technician needed for the setup and maintenance? Are upgrades necessary to keep the technology running over time? Are there power supplies, batteries, tapes, memory cards or other components that will require replacement? Are resources available to install/connect technology at the clinical side and the remote location? Another important aspect for rehabilitation management is to be aware of the knowledge level of the operating clinical staff that will interact with the technology on a daily basis. In addition to being able to use the new technology, the clinicians may also need to be able to do quick repairs or provide help to their clients during remote sessions.

\section{ACCESSIBILITY AND USABILITY}

As noted earlier in our discussion of usability characteristics, the usability of technology may have an impact on how and if a telerehabilitation technology will replace current clinical practice. Brennan and Barker (2008) noted numerous human factors to consider in telerehabilitation -- the age, education, and technology experience of users, and the patients' acquired impairments. Another barrier to technology adoption is the required learning curve for users on both the clinical and remote sides. Users must understand a technology or software and be able to independently adjust settings and maintain/upgrade the technology. Technology abandonment may occur (even in the face of unmet clinical needs), when users decide that a telerehabilitation technology is too difficult to learn or requires high levels of maintenance.

Skill and product experience of a user can affect the ease of use of a TT. Many older Americans are inexperienced in the use of technologies. A study by Rosen (2001) concluded that technical (un)savvyness, hearing and visual problems of elderly patients may have affected the effectiveness of TT used to monitor elderly patients with chronic lung disease living in rural areas. Other aspects that affect the usability of a technology are users' functional and cognitive limitations. Potential users of TTs include the patient, their caregivers, clinicians and physicians, rehabilitation engineers etc. Some of these individuals are on the clinical side, whereas others are on the receiving patient side of the TT. Because there are two or more users involved with the TT, the human interaction system needs to work and be usable from both sides, meaning that both rehabilitation professionals as well as clients need to be able to use the TT (Bashur, 2002). Many consumers of telerehabilitation are in need of services because they have recently experienced a significant change in their functional status, and may be much less capable physically or cognitively of engaging in the use of keyboards or other technology interfaces than they had been before the onset of disability. Any technologies that require the consumer to initiate use or respond will therefore need to address the accessibility issues related to modified or impaired sensation, perception, motor skills, or cognition. Technology usability can be assessed by perform usability studies to directly observe if actual clients encounter difficulties during the setup, installation and general use of the technology.

\section{COST}

Among the variety of devices available for Telerehabilitation, there are affordable technologies such as a computer with an Internet connection and web camera used in combination with readily available conference tools (i-Sight, Skype, MSNmessenger, Adobe Connect, etc.). An example of a high-end, expensive system is a big screen video conference setup using 
special camera equipment and a software based video conferencing program that requires annual updates. If the purpose is to simply communicate and see clients remotely, the first option may be sufficient and cost effective (no phone cost) for individuals who already have a computer setup in their home or clinic.

\section{SYNCHRONY}

Telerehabilitation can replace the need for real time supervision and two sided (face-to-face) communication due to the opportunity for asynchronous data exchange. A neuro psychologist can retrieve data from a PDA that was previously collected and stored by a client in another place and time. Telerehabilitation technologies such as robotic recording devices, wearable technologies (activity monitors), video cams and PDA's can gather data at the clients home, which can later on be transferred by phone, mail, email or web after which it can be accessed and evaluated by a skilled clinician. By disconnecting direct data gathering and communication between the client and clinician, there could also be a change in technology complexity from very complex (e.g., a speech recording device at the client's home wirelessly provides data to a clinician through the web in real time) to simple data collection ( e.g., remote robot and data collection on a USB stick which is emailed to the clinician).

Note that when disconnecting the data collection from the data evaluation portion, there could be a potential risk that data is misinterpreted by the clinician because there is a lack of real time feedback. Therefore, types of applications wherein asynchronized data can be successful include speech therapy, during which the client prerecords speech onto a device residing at their home and the speech-language pathologist listens to the recorded speech at a later time. Clinical applications that may not be suitable for an asynchronized approach include a cognitive assessment of clients with brain injury, because real time client-clinician interaction is important and human interaction and body language need to be observed in real-time.

Successful use of telerehabilitation technology requires that all stakeholders (clinical service providers, clients and caregivers) are able to collectively acquire, install, and use the technology given their capabilities and available technical support. To reduce the chance of abandonment, the technology needs to be affordable over time so that updates, software renewals, and broken parts can be easily upgraded and/or repaired and replaced by those within the organization and/or remote site. It is advisable to select technology with the lowest complexity as well as a technology that can be installed by someone within the organization. The technology should also be selected so that it is widely accessible and usable by a variety of individuals with different backgrounds, experiences, and functional and cognitive abilities.

Seemingly appropriate technology may be abandoned by clinicians and (remote) clients or their caregivers if issues such as complexity, usability, accessibility, installation, and cost are not acknowledged and are not part of the initial technology selection process.

Telerehabilitation Applications

Apart from acquiring, setting-up, using and maintaining telerehabilitation technology, we need to consider the application for which the technology will be used. Each type of clinical application, (e.g., vocational service or occupational therapy follow-up), has its own set of required measures or datasets. For example:

- During a vocational rehabilitation session the therapist may need to see the person's face and study his body language. A system displaying both audio and visual data is needed on both the clinical and remote side.

- During an occupational therapy consult, the therapist may need to obtain a client's weekly activity level to track overall fitness. A wearable and/or wheelchair mounted accelerometer can measure bodily and wheelchair activity and data can be emailed to the therapists for interpretation.

Winters previously established a specific set of services (Table 2). By slightly altering this list, the services can be paired and associated to the available telerehabilitation technologies (Table 4).

Table 4: Matching technologies and clinical applications.

Matching Technologies and Clinical Applications

Service/Application: Education

Character: Independent

Data requirement: Textual/Audio/Visual

Applicable Technology: Lesson on Tape, Web Courses, Video Courses

Service/Application: Physical assessment/evaluation

Character: Team

Data requirement: $\quad$ Audio/Visual/Haptic

Applicable Technology: Video conferencing, Robotics

Service/Application: Cognitive assessment/evaluation

Character: Team

Data requirement: Textual/Audio/Visual

Applicable Technology: Video Conferencing 


\section{DISCUSSION}

Beyond the technical information and suggested protocols for analysis of TT usability described above, pragmatic issues also affect successful implementation of TT in rehabilitation settings. For most planned telerehabilitation services, TT offers a means of replacing face-to-face (FTF) services with some form of remote service. This can only occur successfully if there is some value-added that accrues through the use of TT over and above the continued provision of face-to-face services. While there are numerous potential incentives to TT such as decreased travel time, cost reductions, access to services otherwise unavailable, or access to specialty knowledge, there will also be some minimum threshold to cross before an individual chooses to use TT over FTF services. The factors involved in crossing this threshold will differ from person to person, but may include the perceived difficulty of TT use, perceived or real trade-off of benefits from TT compared to loss of FTF experience, and the learning curve of using TT. In particular, the issue of having repeated successful experiences using TT before integrating telerehabilitation services may pose a significant obstacle to crossing the TT threshold. Even as technically proficient humans using email and the Internet extensively, we continue to engage in direct, real-time human contact for the vast majority of our successful interpersonal communications.

Improving TT's should acknowledge (dis)ability related issues and human factors as described in this paper. To enhance the usability and reduce the adoption threshold, technologies should be designed with the end-user in mind. Universal design principles may be one approach to design TTs. This type of approach incorporates the need for technology to be reliable, approachable and usable by all users, with a clear incentive for use over current FTF interactions, and with minimal training required.

More research is needed to educate clinicians and potential users on the range of technologies that can be used for telerehabilitation.

\section{REFERENCES}

Bashshur, R.L. (2002). Telemedicine and health care. Telemedicine Journal and e-health, 8(1), 5-12.

Benedixen, R.M., Levy, C., Lutz, B.J., Horn, K.R., Chronister, K., \& Mann, W.C. (2008). A telerehabilitation model for victims of polytrauma. Rehabilitation Nursing, 33(5), 215-220.

Brennan, D.M., \& Barkder, L. (2008). Human factors in the development and implementation of telerehabilitation systems. Journal of Telemedicine and Telerate, 14, 5558.

Brewer, B., McDowell, S.K., Worthen-Chaudhari, L.C., (2007). Poststroke upper extremity rehabilitation: a review of robotic systems and clinical results. Topics in Stroke Rehabilitation, 14(6) 22-44.

Carnigan, C.R., \& Krebs, H.I. (2006). Telerehabilitation robotics: bright lights, big future? Journal of Rehabilitation Research and Development, 43(5), 695710.

Darkins, A., Cruise, C., Armstrong, M., Pters, J., \& Finn, M. (2008). Enhancing access of combat-wounded veterans to specialist rehabilitation services: the VA polytrauma telehealth network. Archives of Physical Medicine \& Rehabilitation, 89, 182 - 187.

Diamond, B.J., Shreve, G.M., Bonilla, J.M., Johnston, M.V., Morodan, J., \& Branneck, R. (2003). Telerehabilitation, cognition, and user-accessibility. NeuroRehabilitation, 18, 171-177.

Girard, P. (2007). Military and VA telemedicine systems for patients with traumatic brain injury. Journal of Rehabilitation Research and Development, 44(7), 1-10.

Koocher, G.P. (2007). Twenty-first century ethical challenges for psychology. American Psychologist, 62(5), 375-384.

Lorig, K., Ritter, P.L, Laurent, D.D., \& Plant, K. (2006). Internet-based chronic disease self-management: a randomized trial. Medical Care, 44(11), 964-971.

Rosen, E. Twenty minutes in the life of a tele-home healthcare nurse. (2001). Telemedicine Today. [cited 2004 June 9].

Story M.F. Maximizing usability: the principles of universal design. Assistive Technology 10, No. 1 (1988): 4-12.

Theodorus, D., \& Russell, T. (2008). Telerehabilitation: current perspectives. Studies in Health Technology \& Informatics, 131, 191-208.

Tula, B., Chatterjee, S., \& Laxminarayan, S. (2005). A taxonomy of telemedicine efforts with respect to applications, infrastructure, delivery tools, type of setting, and purpose. Proceedings of the 38th Hawaii International Conference on System Sciences - 2005.

Winters, J.M. (2002) Telerehabilitation research: Emerging opportunities. Annual review of Biomedical Engineering, 4, 287-320.

Table 1 (c)Copyright 1997 North Carolina State University, The Center for Universal Design 
Corresponding address: Michael Pramuka PhD, University of Pittsburgh

Department of Rehabilitation Science and

Technology

5036 Forbes Tower, Pittsburgh, PA, 15260, USA,

Email: mpramuka@pitt.edu

Linda van Roosmalen PhD,

University of Pittsburgh

Department of Rehabilitation Science and Technology

5036 Forbes Tower, Pittsburgh, PA, 15260, USA,

Email: Ivanroos@pitt.edu

This document is a draft intended for use by

participants in the Rehabilitation Engineering

Research Center on Telerehabilitation's State of

the Science Conference to be held November

17-20, 2008. The document may not be distributed

and should be discarded after the conference.

A final draft will be developed in response to the

discussions taking place during the conference.

The paper will be published in final form in the

April 2009 issue of the International Journal of

Telerehabilitation. We look forward to your active

participation in the conference and welcome any

comments you have related to the information

presented here. 